\title{
Present and Future Challenges in Food Analysis. Foodomics
}

Virginia García-Cañas ${ }^{\psi}$, Carolina Simó ${ }^{\psi}$, Miguel Herrero, Elena Ibáñez, Alejandro Cifuentes ${ }^{*}$

Laboratory of Foodomics, CIAL (CSIC), Nicolas Cabrera 9, 28049 Madrid, Spain.

*Author for the correspondence: $\underline{\text { a.cifuentes @ csic.es }}$

${ }^{\psi}$ These two authors contribute equally to this work 


\section{Summary}

The state-of-the-art of food analysis at the beginning of the $21^{\text {st }}$ century is presented in this work, together with its major applications, current limitations and present and foreseen challenges.

\section{Food Analysis: The Basis}

The development and application of analytical methods and techniques in food science has grown parallel to the consumers concern about what is in their food and the safety of the food they eat. To give an adequate answer to the raising consumers' demands, food analysts around the world are facing increasingly complex challenges that require using the best available science and technology. A good portion of this complexity is due to the so-called Globalization and the movement of food and related raw materials worldwide, which are generating contamination episodes that are becoming also global. An additional difficulty is that many products contain multiple and processed ingredients, which are often shipped from different parts of the world, and share common storage spaces and production lines. As a result, ensuring the safety, quality and traceability of food has never been more complicated and necessary than today ${ }^{1}$.

The first goal of food analysis has traditionally been, and still is, to ensure food safety. To meet this goal, food laboratories are being pushed to exchange their classical procedures for modern analytical techniques that allow them to give an adequate answer to this global demand. Besides, the new European regulations in the European Union countries (e.g., Regulation EC 258/97 or EN 29000 and subsequent issues), the Nutrition Labeling and Education Act in the USA, and the Montreal Protocol have had a major impact on food laboratories. Consequently, more powerful, cleaner and cheaper analytical procedures are now required by food chemists, regulatory agencies and quality control laboratories. These demands have increased the need for more sophisticated instrumentation and more appropriate methods able to offer better qualitative and quantitative results while increasing the sensitivity, precision, specificity and/or speed of analysis. $^{2}$

Apart of these essential considerations, there are also a large number of food properties for which Analytical Chemistry will play a crucial role. Just to mention a few, the identification of the effect of food production, processing, preparation, and use on nutrient content, toxic contaminant generation, and inactivation of naturally occurring toxins; the compliance with food and trade laws ensuring food safety and traceability; the detection of adulteration and 
product tampering; the characterization of chemical composition of foods; the study of food rheology, morphology, structure or surface; the analysis of physical, physicochemical, thermal or microbiological properties; the evaluation of sensory characteristics, etc. These properties will have a critical influence on food safety, quality, processing and acceptance. ${ }^{3}$

Currently, there is also a general trend in food science towards the connection between food and health. Thus, food is considered today not only a source of energy but also an affordable way to prevent future diseases. The number of opportunities (e.g., new methodologies, new generated knowledge, new products, etc) derived from this trend are impressive and it includes, e.g., the possibility to account for food products tailored to promote the health and well-being of groups of population identified on the basis of their individual genomes. The introduction in this area of research of advanced "omics" approaches such as Foodomics ${ }^{4}$ have made possible that food scientists can face problems unthinkable few years ago. However, to achieve these goals, researchers involved in modern food science need an adequate background on advanced analytical tools in order to extract all the potential from these new methodologies. Usually, a sine qua non condition is to work within multidisciplinary teams in order to be able to face the huge complexity of the problem and to handle the generated results in a rational way.

Following it will be shown that food analysis is, nowadays, one of the most important application areas of Analytical Chemistry. Namely, the main analytical techniques employed in food analysis at the beginning of the $21^{\text {st }}$ century will be presented together with their main application areas and current difficulties, concluding with an outlook on some anticipated future challenges in this crucial and hot area of research.

\section{Analytical Chemistry and Food Analysis in the $21^{\text {st }}$ Century.}

A description of the huge number of analytical techniques commonly used in food analysis is out of the scope of this work. Just to mention a few ones, analytical techniques typically used in food analysis can be classified in: i) spectroscopic as mass spectrometry (MS), nuclear magnetic resonance (NMR), infrared (IR), atomic spectroscopy (AS), fluorescence, etc; ii) biological as polymerase chain reaction (PCR), immunological techniques, biosensors, etc.; iii) separation as high performance liquid chromatography (HPLC), gas chromatography (GC), capillary electrophoresis (CE), supercritical fluid chromatography (SFC), etc.; iv) sample preparation as solid phase extraction (SPE); supercritical fluid extraction (SFE); headspace (HS); flow injection analysis (FIA); purge and trap (PAT); pressurized liquid extraction (PLE); microwave assisted extraction (MAE); automatic thermal desorption (ATD), etc; v) electrochemical; vi) 
hyphenated techniques; etc. The number of techniques in food analysis is even higher if the subdisciplines under the above techniques are considered.

To help summarizing the large number of analytical techniques used and topics addressed in food analysis, Table S-1 is included as Supplementary Information to describe all the works published in the last three years (i.e., 2009-2011) as review papers, books and book chapters (more than 160) on different food analysis subjects. Moreover, Figures 1 and 2 provide information on the number of works published in the period 2001-2011 found through a search in the database Food Science and Technology Abstracts (FSTA) using as key terms the names of the analytical technique indicated in each case. There are some important issues that can be concluded from Figures 1 and 2 when they are compared to similar figures published by our group at the end of the $20^{\text {th }}$ century summarizing the works published on food analysis in the period 1990-2000. ${ }^{5}$ The most important trend is the huge increase in biological and sample preparation techniques as compared with the previous period and the important decrease in the use of radiochemical and thermal techniques, probably due to the specific information that those techniques provide and the need for high-throughput techniques widely based on new and advanced technologies able to provide with more information of better quality. Thus, it is not strange that techniques such as thermal and radiochemical have decreased by half (compared to the previous period) and others such as spectroscopic, biological, and sample preparation techniques have increased two, three and four times, respectively. Other well established techniques such as separation techniques continue to be used in a high extend but nowadays they are not the most widely used (as in the period 1990-2000), since spectroscopic techniques have gained importance and are at present the most extensively used in food analysis. In fact, the detection and content of a number of food constituents, as well as the study of food properties, may be achieved by measuring the interaction of electromagnetic radiation (absorption in the visible, infrared, fluorescence, Raman, etc.) with food. Thanks to new instrumental developments of spectroscopic techniques together with multivariate chemometric methods, that are appropriate and useful for the evaluation of fluorescence or infrared spectra exhibiting slight differences such as the ones recorded on food products, it has been possible to develop prediction models. Recently, imaging technology such as confocal laser scanning microscopy or hyperspectral imaging coupled to image analysis techniques has successfully been used to study highly heterogeneous food products. Indeed, image analysis techniques such as mathematical morphology or image texture analysis make it possible to quantify structures in the images and to show the influence of different manufacturing processes on the protein network microstructure of foods. ${ }^{6}$ On the other hand, the important increase in the use of spectroscopic techniques in food analysis might be due to the high number of new applications of NMR, fluorescence, IR, etc. that can be, in the case of NMR, attributed to the need for 
unambiguous identification of unknown compounds with biological properties, metabolites, etc., that probably has contributed to the implementation of this technique with numbers quite close to well established techniques such as fluorescence or even MS.
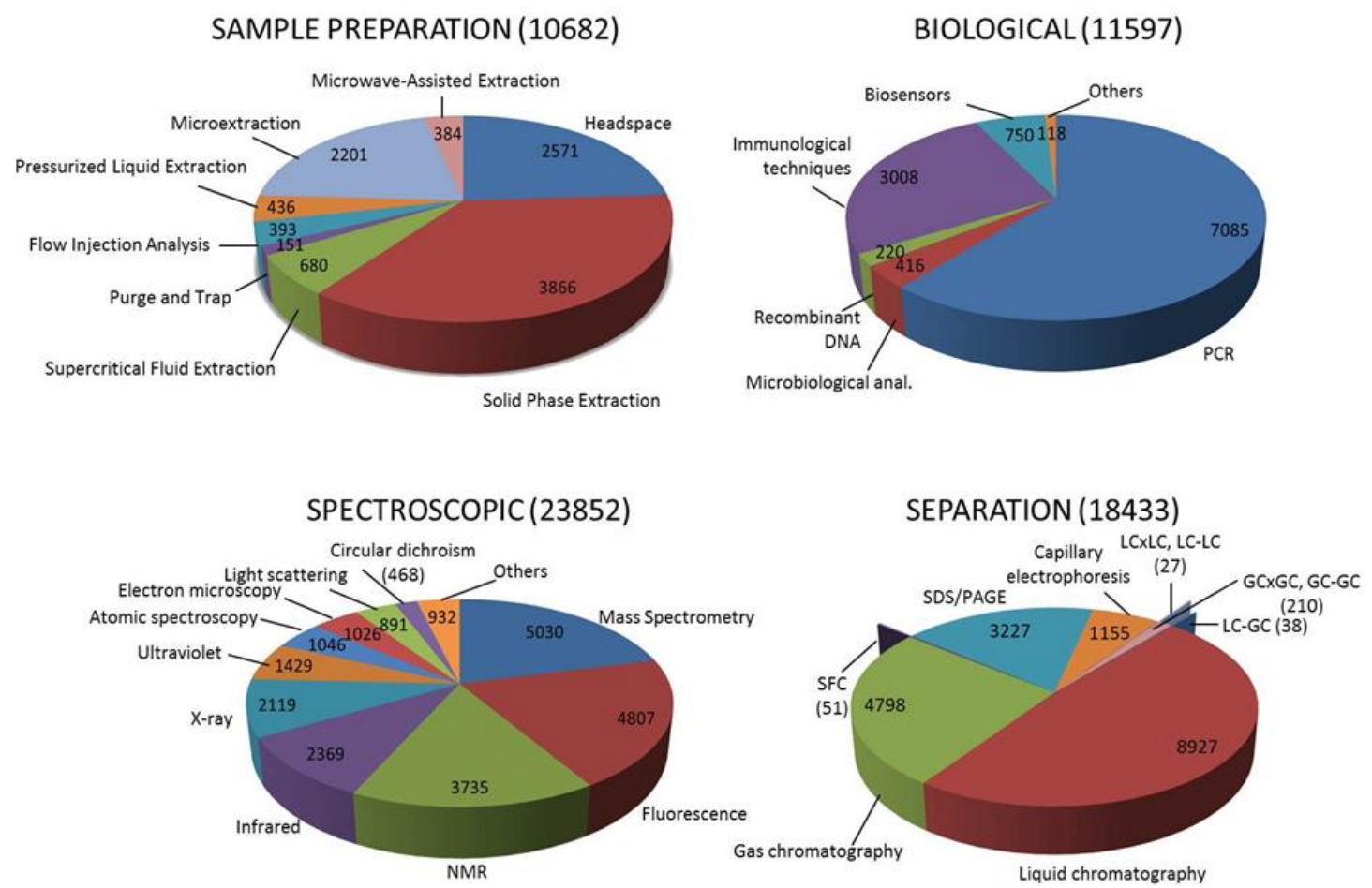

Figure 1. Sample preparation, biological, spectroscopic and separation techniques used in food analysis and the number of citations in FSTA database in the period 2001-2011. Within biological techniques, the group "others" includes: radioimmunoassay and enzymatic analysis. The group "others" in spectroscopic techniques includes: raman (402), electron spin resonance (366), dielectric spectroscopy (57), refractometry (54), polarimetry (38), chemiluminiscence (15), photoacustic (0). 
ELECTROCHEMICAL (1883)

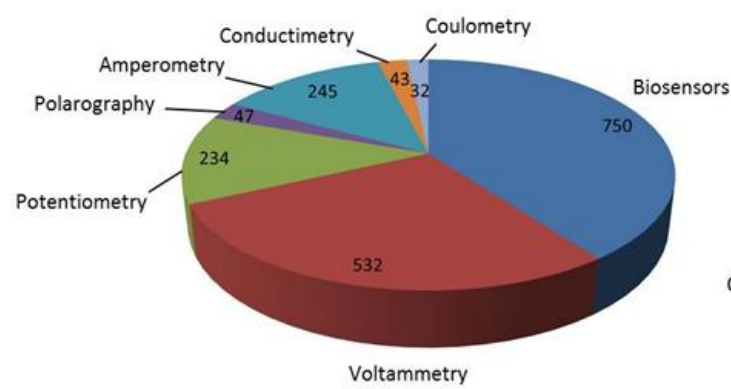

RADIOCHEMICAL (300)

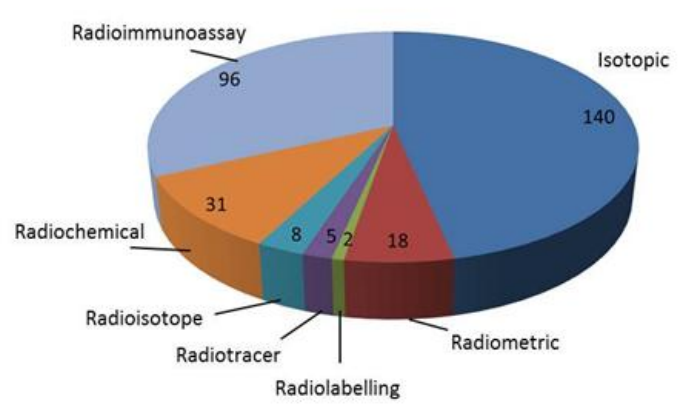

RHEOLOGICAL (943)

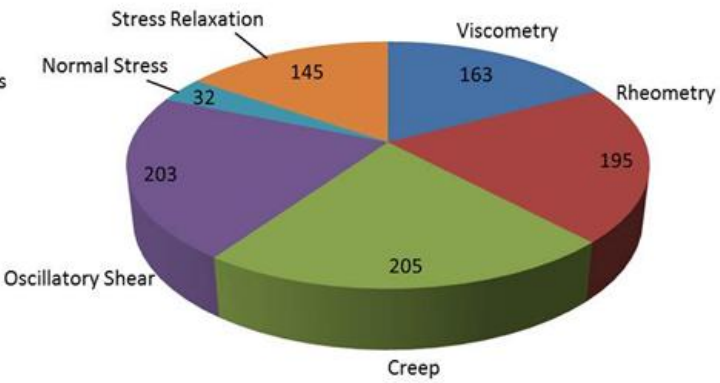

THERMAL (593)

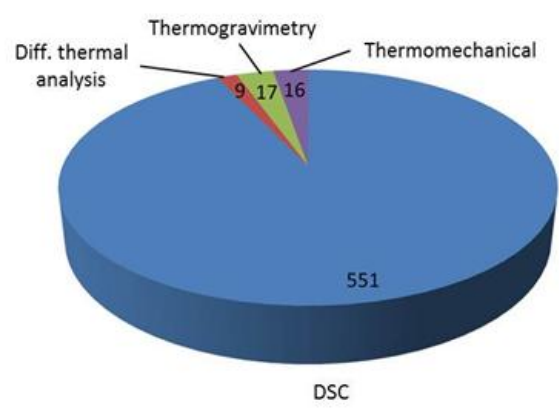

Figure 2. Electrochemical, rheological, radiochemical and thermal techniques used in food analysis and the number of citations in FSTA database in the period 2001-2011.

Not surprising is the huge increment in the use of biological techniques; these techniques, based on the use of living organisms or some of their products such as enzymes, antibodies, DNA, etc. to identify and analyze foods, have multiply by three their use in food analysis; one technique, PCR, constitutes a $60 \%$ of the total applications in biological techniques being around double of the total number of biological techniques used in the previous period. This huge increase in the use of PCR can be attributed to the different steps taken to overcome some of the main difficulties of the technique, related to the quality and amount of DNA extracted; at present, new instruments and new standardized protocols, for an important number of samples, make PCR a widely used technique worldwide and can be found in almost all laboratories working in food analysis.

As for the distribution and importance of the separation techniques, LC and CE applications have raised mainly due to the new developments for reducing analysis time while keeping resolution and efficiency (UPLC, on-chip CE, monolithic columns), the new separation mechanisms (HILIC, etc,), and the use of MS as a routine detector for LC and CE. On the other hand, GC is keeping similar numbers as previously, showing its importance for certain applications. Lastly, the important increase in hyphenated separation techniques (LCxLC, LC- 
LC, GCxGC, GC-GC, LC-GC), supports the theory that more information is needed to be able to decipher the wide complexity of food samples and their real effects in human health. In fact, multidimensional chromatography has become an interesting alternative to analyze complex samples also in food analysis in a situation in which technological improvements, such as new column technologies, seem to be reaching their maximum level. Thus, peak capacity enhancement achievable by multidimensional chromatography is by far higher than the obtained after improving by any mean one-dimensional separations. Multidimensional chromatography allows combination of two or more independent or nearly independent separation steps, increasing significantly the separation power of the corresponding one-dimensional techniques and, therefore, the physical separation of compounds in complex samples. Although the coupling between different chromatographic separations is not new, the technological development has led, above all, to the increase of comprehensive applications in which the whole sample is analyzed in two different, independent dimensions, reducing the sample preparation steps. The number of applications regarding the use of such comprehensive techniques increases every year also in the food analysis domain and it is expected to keep growing. ${ }^{7}$

Special attention has to be also paid to the important (around 4 times) increase in the use of sample preparation techniques in food analysis. Advances in sample preparation aim to minimise laboratory solvent use and hazardous waste production, save employee labour and time, and reduce the cost per sample, while improving the efficiency of the analyte isolation. At present, new green sample preparation methods such as SFE and subcritical water extraction (SWE, also called accelerated solvent extraction) are among the more promising processes in food science, not only in food analysis but also for obtaining new functional food ingredients. ${ }^{8}$ These extraction techniques based on pressurized fluids provide higher selectivities, shorter extraction times and are environment-friendly. The total numbers for these techniques in the period 2001-2011 reached over 1500 publications, compared to around 300 at the end of $20^{\text {th }}$ century; one interesting example is, for instance, PLE, that did not appear in the previous period and is nowadays the second more important "green" sample preparation technique used in food analysis, only surpassed by SFE. Also, different modes of liquid-phase microextraction (LPME) such as single drop microextraction, dispersive liquid-liquid microextraction and hollow fiberLPME are being increasingly applied for the extraction of both inorganic and organic analytes from different matrices in food analysis, due to their advantages over conventional extraction procedures in terms of simplicity, effectiveness, rapidity and low consumption of organic solvents. ${ }^{9}$ Another important observation, when comparing the numbers and distribution of sample preparation and separation techniques, is the increasing use of SPE that somehow runs parallel to the use of liquid chromatography for which new separation mechanisms, new 
applications and new approaches have been developed in these last 10 years. It is interesting also to observe that the number of applications of, for instance, SPME have come close to other well-established techniques such as headspace; the expansion of SPME since the last period considered can be understood due to the easiness of use, the huge range of applicability of fibers and coatings and the new modalities developed that have widen the range of applicability.

Other more specific application areas in food analysis have also seen a great development as a result of the combination of several analytical advances that have been put together. This is the case of the analysis of the volatile fraction of foods, which is known to have a crucial effect on food quality and acceptance. The study of the volatile fraction of food or beverage requires analytical methods and technologies able not only to evaluate its composition exhaustively but also to monitor variations of its profile and to detect trace components characterizing the food being investigated. The strategies of analysis have changed significantly over the last 15-20 years because of the introduction of new approaches, in particular: (i) solventless sample preparation techniques; (ii) fast GC and related techniques; (iii) new analytical techniques, such as comprehensive GC; (iv) new operative strategies based on approaches developed for other fields and applied to food analysis; and (v) data elaboration strategies producing a higher level of information. ${ }^{10}$ Chiral analysis has also seen an important growing in food analysis, since chiral methods can be used to study and characterize foods and beverages through the enantiomeric separation of different food compounds such as amino acids, pesticides, polyphenols, etc. ${ }^{11}$ Another example is the investigation on food texture in which physical characteristics perceived by the senses are investigated. Research in this area has evolved tremendously in the last decade based on multidisciplinary approaches that encompass chemistry, physics, physiology, and psychology, to study fracture of food, the sounds it makes during biting and chewing, its microstructure, muscle movements during mastication, swallowing and acceptability, etc. ${ }^{12}$ The determination of contaminants in foods is a must for ensuring that human exposure to noxious residues though diet does not exceed acceptable levels for health. Consequently, robust analytical methods are continuously under development in order to improve recovery rates, quantification limits, time of analysis, or to reduce matrix effects. ${ }^{13}$ Interestingly, nowadays, method validation is also required for carrying out both research and monitoring programs, and thus for defining limitations and supporting enforcement of regulations. ${ }^{14}$

It is also noteworthy to describe how MS has evolved in the last years in food analysis. During the last decade, MS has tended to be used largely for direct identification and quantification of food compounds typically coupled to other separation techniques like LC and, in a less extent, CE. Single quadrupole MS has been restricted to screening purposes since these instruments do 
not meet the more recent criteria set by the EU, especially those regarding the requested number of identification points. As a result, tandem-MS has become a general tool for identification and quantification of analytes (mainly contaminants) in food analysis. The enhanced selectivity afforded by tandem-MS detection may also contribute to the simplification of the extraction procedure, if attention is paid to ion suppression phenomena. At this point, the use of triple quadrupole, ion trap and more recently time of flight MS analyzers coupled to uni- or bidimensional separation techniques have been widely reported in the scientific literature in food analysis. ${ }^{15}$ It is expected that new developments on ionization techniques prior to MS analysis can make even broader its application in food analysis including new omics applications. ${ }^{16}$ Proteomics and metabolomics represent powerful analytical platforms to acquire more detailed and complete information on food composition even beyond the traditional food component analysis. This comprehensive knowledge of biochemical composition of foods will provide a better understanding of metabolic networks allowing food research community for a better insight of the molecular basis of important food characteristics such as flavour, colour, texture, aroma, added-value nutrition, etc. ${ }^{17}$ In this context, metabolomics (via GC-MS, LC-MS, CE-MS or NMR) has potential to add significant value to crop and food science, raw material quality and safety, food storage, shelf-life and post-harvest processing. ${ }^{18}$ The ability of different transcriptomic, proteomic and metabolomic approaches has already been shown to assess food safety and quality at every stage of production to ensure food safety for human consumption. ${ }^{19}$ They are also valuable tools to distinguish between similar food products and to detect food frauds (adulteration, origin, authenticity, etc), food-borne pathogens, toxic species, food allergens, etc. For instance, in the context of food safety, several DNA microarray chips have already been developed for the detection of food-borne pathogens, toxigenic microorganisms, genetically modified (GM) organisms analysis, etc. Proteomic and metabolic changes also occur during crops growing conditions, food processing/preparation (fermentation, baking, boiling, etc), food conservation/storage (freezing, smoking, drying, etc). These tools have already been demonstrated to be very useful for getting a deeper understanding of molecular details of foods and food related matrices ${ }^{20}$ including the analysis of GM foods ${ }^{19}$. In this later case, the use of omics approaches able to provide useful fingerprints of GM foods (e.g., for GM detection, composition monitoring, traceability, study of unintended modifications, labelling issues) has already been recommended by the European Food Safety Authority. ${ }^{21}$

\section{Present and Future Challenges in Food Analysis.}

In spite of the huge number of analytical developments and applications seen in food analysis, there are still a good number of issues that need to be improved in this hot area of research. For instance, still hundreds of foodborne infection cases occur around the world, and up to one third 
of the population in industrialized nations suffers from foodborne illness each year. Regarding pathogens detection in foods, microbiologists have developed over the last decades reliable culture-based techniques. Although these methods are considered to be the "gold-standard," they remain cumbersome and time consuming. The introduction of genetic-based technologies made feasible developing sensitive and specific screening tests for the detection of microbial pathogens. Microarray-based technologies represent an advance in nucleic acid testing methods whose main features include miniaturization, ability to parallelize sample processing, and ease of automation. ${ }^{22}$ Besides, the high number of genetic information already available allows reaching a resolution below the species level, being able to discriminate among microbial strains, thanks to the careful choice of variable genomic regions. Despite the advent of these rapid detection methods based on molecular techniques (or immunoassays), it is suggested that reduction and/or elimination of cultural enrichment will be essential in the quest for truly realtime detection methods. As such, there is an important role for the so-called pre-analytical sample processing that in this case would include bacterial concentration and purification from the sample matrix as a step preceding detection. ${ }^{23}$ In this regard, one analytical challenge that still remains in food safety is to present reliable results with respect to official guidelines, as fast as possible without impairing method properties such as recovery, accuracy, sensitivity, selectivity, and specificity. ${ }^{24}$

More suitable analytical techniques are still required by consumer protection and law enforcement for the detection of allergens in foods. Food allergy is an important issue in food analysis because minute amounts of the allergen can have critical consequences in sensitized persons, what has brought about very demanding requirements on hygiene and legal regulations imposed on the food industry. Immunological methods are currently preferred followed by confirmatory methods. The determination of allergenic proteins by LC and MS has greatly advanced in recent years and it is now frequently used for the identification and quantitation of food allergens. ${ }^{25}$ In spite of these advances, confirmatory alternatives are still needed able to face other additional problems originated e.g., by food matrix interferences or food processing, which may not influence allergenicity but do impair allergen detection.

Miniaturization of analytical systems will keep under development including newly emerging technologies able to offer platforms with greater automation and multiplexing capabilities than traditional biological binding assays. These multiplexed bioanalytical techniques are expected to provide control agencies and food industries with new possibilities for improved, more efficient monitoring of food and environmental contaminants. In this regard, developments in planararray and suspension-array technologies have demonstrated their potential in detecting pathogens, food allergens and adulterants, toxins, antibiotics and environmental contaminants. ${ }^{26}$ 
In this context, microfluidics technology has also shown interesting applications for food analysis, although more effort has to be put on the development of multi-purpose microfluidic platforms that integrate multiple unit operations for real food sample analysis. ${ }^{27}$ Miniaturized systems and their applications are expected to keep growing in food analysis.

Regarding multidimensional chromatography methods, from a technical point of view, some problems inherent to the connection of the two systems still persist, for instance related to the relatively costly operation conditions in $\mathrm{GC} \times \mathrm{GC}$ or the loss in sensitivity in LCxLC. In the coming years, new solutions should appear in order to facilitate these couplings as well as to further increase the orthogonality of the systems and, consequently, their separation power and applications in food analysis. Keeping the cost of analysis as low as possible should be also a priority when designing efficient and new comprehensive GC and SFC modulators. Besides, the development of on-line sample preparation steps in multidimensional systems can be also expected: Moreover, the extended use of powerful MS detectors would enhance even more the applications and identification power of these techniques in food analysis. ${ }^{7}$ An important point to consider is that nowadays these multidimensional techniques require dedicated laboratories, equipment and highly-trained personnel, till they can offer simpler and more rapid analysis.

Food-associated viruses are of emerging importance is food analysis as causative agents of gastrointestinal diseases and hepatitis. Due to the development of molecular biological methods the detection of noroviruses, rotaviruses, hepatitis A viruses and hepatitis B viruses and other relevant viruses in different food matrices is now feasible. However, the designated methods need to be improved since their efficiency varies considerably depending on the method used, the food matrix and the type of virus. Standardized test procedures are needed for a realistic comparison of the existing methods. ${ }^{28}$

The variety of toxic residues in food is continuously increasing as a consequence of industrial development, new agricultural practices, environmental pollution, and climate change. This increasing is bringing about the development of everyday more powerful, sensitive and fast analytical methodologies able to detect emerging contaminants in food like industrial organic pollutants, nanomaterials, pharmaceutical residues antibiotics and coccidiostats or emerging groups of marine biotoxins. ${ }^{29}$

Nanotechnology and nanomaterials have remarkable potential to enhance the food supply through novel applications, including nutrient and bioactive absorption and delivery systems; microbial, allergen, and contaminant detection and control; food packaging properties and performance; and improved colors and flavors. Based on these multiple applications, exposure 
to nanomaterials in the human food chain may occur not only through intentional uses in food manufacturing, but also via uses in agricultural production and carry over from use in other industries. New analytical methods are, therefore, needed to fully detect and characterize nanomaterials incorporated into foods and in other media. Moreover, there is also a need for additional toxicology studies on different types of nanomaterials to understand how they can affect food safety. ${ }^{30}$

Finally, a clear trend is the implementation of Green Analytical Chemistry also in food analysis laboratories, understanding Green Analytical Chemistry as "the use of analytical chemistry techniques and methodologies that reduce or eliminate solvents, reagents, preservatives and other chemicals that are hazardous to human health or the environment and that may also enable faster and more energy-efficient analysis without compromising performance criteria". In this definition it is clear that the hazard is to be reduced but keeping (o even improving) the analysis in terms of performance. Several approaches such as those concerning the greening of sample preparation techniques (with the use of new green solvents, miniaturization or employment of solventless techniques) and the combination with new (and cleaner) separation techniques and chemometrics, will greatly contribute reach the goals of this new Green era. ${ }^{31}$

\section{Foodomics. A New Discipline for a New Food Era.}

One of the main challenges in food analysis will be to improve our limited understanding of the roles of food compounds at molecular level (i.e., their interaction with genes and their subsequent effect on proteins and metabolites) for the rational design of strategies to manipulate cell functions through diet, which is expected to have an extraordinary impact on our health. In this context, Foodomics has been defined as a new discipline that studies the food and nutrition domains through the application of advanced omics technologies to improve consumer's wellbeing, health, and confidence. ${ }^{4}$ Thus, Foodomics is presented as a global discipline in which food (including nutrition), advanced analytical techniques (mainly omics tools), and bioinformatics are combined. The development of genomics, transcriptomics, proteomics and metabolomics has given rise to extraordinary opportunities for increasing our understanding about different issues that can now be addressed by Foodomics. Just to name a few: (i) to understand the biochemical, molecular and cellular mechanisms that underlies the beneficial or adverse effects of certain bioactive food components following nutrigenomic approaches; ${ }^{32}$ (ii) to understand the gene-based differences among individuals in response to a specific dietary pattern following nutrigenetic approaches; ${ }^{33}$ (iii) to know the identity of genes that are involved in the previous stage to the onset of the disease, and therefore, possible molecular biomarkers; ${ }^{34}$ (iv) to determine the effect of bioactive food constituents on crucial molecular pathways; ${ }^{35}$ (v) 
to establish the global role and functions of gut microbiome, a topic that is expected to open an impressive field of research ${ }^{36}$ (vi) to understand the stress adaptation responses of food-borne pathogens to ensure food hygiene, processing and preservation; ${ }^{37}$ (vii) to investigate the use of food microorganisms as delivery systems including the impact of gene inactivation and deletion systems; (viii) to carry out the investigation on unintended effects in genetically modified crops $;^{38}$ (ix) the comprehensive assessment of food safety, quality and traceability ideally as a whole $;^{39}(\mathrm{x})$ to understand the molecular basis of biological processes with agronomic interest and economic relevance, such as the interaction between crops and its pathogens, as well as physicochemical changes that take place during fruit ripening, ${ }^{40}$ (xi) to fully understand postharvest phenomena through a global approach that links genetic and environmental responses and identifies the underlying biological networks. In this regard, it is expected that the new omics technologies combined with systems biology, as proposed by Foodomics, can lead postharvest research into a new era. ${ }^{40}$

It is now well-known that health is heavily influenced by genetics. However, diet, lifestyle and environment can have a crucial influence on the epigenome, gut microbiome and, by association, the transcriptome, proteome and, ultimately, the metabolome. When the combination of genetics and nutrition/lifestyle/environment is not properly balanced, poor health is a result. Foodomics is a major tool for detecting small changes induced by food ingredient(s) at different expression levels. A representation of an ideal Foodomics strategy to investigate the effect of food ingredient(s) on a given system (cell, tissue, organ or organism) is shown in Figure 3. Following this Foodomics strategy, results on the effect of food ingredient(s) at genomic/transcriptomic/proteomic and/or metabolomic level are obtained, making possible new investigations at molecular level on food bioactivity and its effect on human health. The interest in Foodomics also coincides with a clear shift in medicine and biosciences toward prevention of future diseases through adequate food intakes, and the development of the socalled functional foods. In this regard, it has been mentioned that it is probably too early to conclude on the value of many substances for health, and the same can apply to other health relationships that are still under study. Thus, Foodomics could help to overcome these limitations. To achieve this goal it will be necessary to carry out more studies to discover more polymorphisms of one nucleotide, to identify genes related to complex disorders, to extend the research on new food products and to demonstrate a higher degree of evidence through epidemiological studies based in Foodomics that can lead to public recommendations. Moreover, in spite of the significant outcomes expected from a global Foodomics strategy, practically there are no papers published in the literature in which results from the three expression levels (transcriptomics, proteomics and metabolomics) are simultaneously presented and merged. Figure 4 shows the results from a global Foodomics study on the chemopreventive 
effect of dietary polyphenols against HT29 colon cancer cells, ${ }^{41}$ presenting the genes, proteins and metabolites identified (after transcriptomic, proteomic and metabolomic analysis) that are involved in the principal biological processes altered in HT29 colon cancer cells after the treatment with rosemary polyphenols. In order to demonstrate all its value, Foodomics still needs to be translated to methods or approaches with medicinal impact, e.g., through the socalled personalized nutrition. In this regard, data interpretation and integration when dealing with such complex systems is not straightforward and has been detected as one of the main bottlenecks.

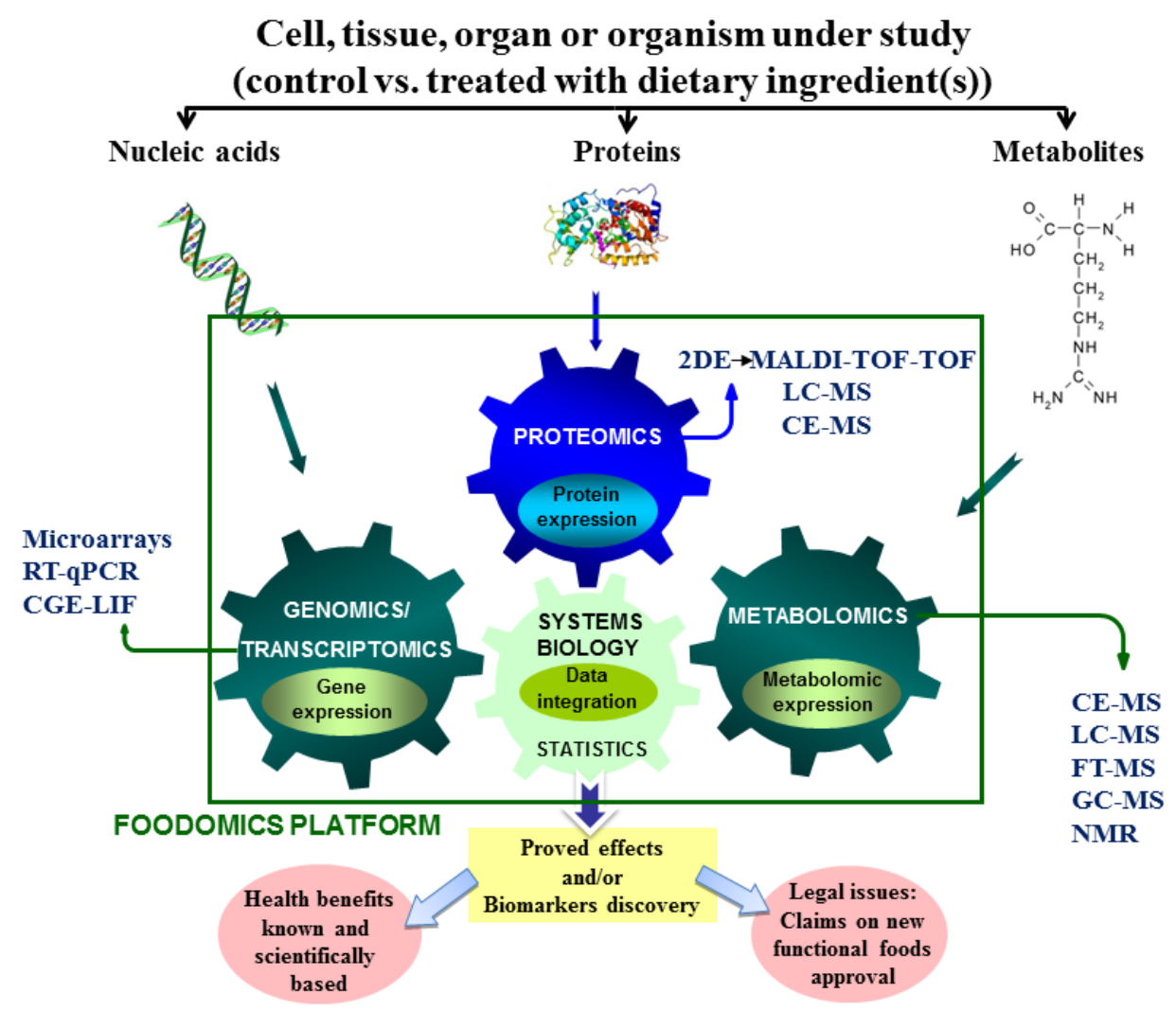

Figure 3. Ideal Foodomics platform to investigate the health benefits from dietary constituents on a given biological system (cell, tissue, organ or organism), including analytical methodologies used and expected outcomes. 


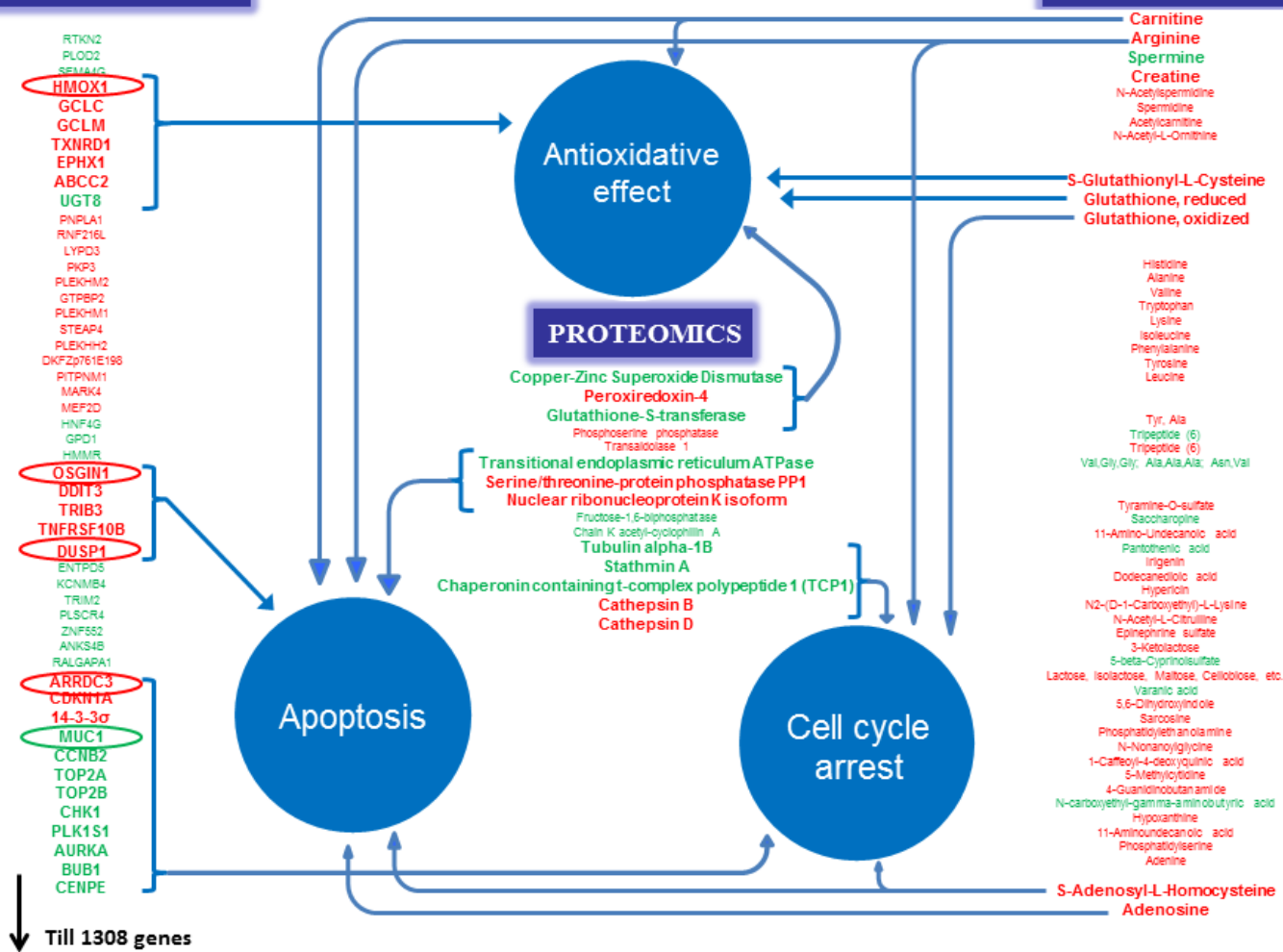

Figure 4. Foodomics identification of the genes, proteins and metabolites involved in the principal biological processes altered in HT29 colon cancer cells after their treatment with rosemary polyphenols. In red: up-regulated; in green: down-regulated. Modified from reference 41.

Foodomics tools will also have to overcome many limitations for optimal implementation in food analysis. For transcriptomics, the high background noise that hinders the detection of low signals (i.e., low signal-to-noise ratios) and the efficiency and specificity of the hybridization probes have to be improved in DNA microarrays. New developments will probably include the establishment of routine data analysis methods and increase in the numbers and lengths of sequence reads as well. It is also expected that the cost of these analyses will continue decreasing in the near future, allowing new applications and extensive use of these technologies in Foodomics research.

In proteomics, MS alone or combined with 2D-electrophoresis, liquid chromatography and capillary electrophoresis have become the most used methodologies. There is an evident need of developing improved or alternative technologies (e.g., protein microarrays) to become into a reality the routine analysis for proteome research, including improvements in the resolution of peptides to provide increased protein coverage. Apart of the everyday more sophisticated sample treatments and separation techniques, MS will keep essential for the systematic 
investigation in proteomics. In this sense, conventional mass spectrometers are giving way to the more sophisticated and compact mass spectrometers, most of them hybrid instruments in a combination of two or more analyzers. As can be deduced from the low number of proteomic applications in Foodomics studies, it is expected that new innovations in proteomic technology will help proteomic profiling to become standard practice also in Foodomics.

A great advance in metabolomics is expected with the incorporation of new MS interfaces for which nearly no sample preparation is needed ${ }^{42}$ and the use of MALDI-imaging mass spectrometry (MALDI-MSI) to analyze from metabolites to proteins at both the tissue and the single-cell level, obtaining information regarding the spatial distribution of specific molecules. ${ }^{43}$ Improvements to both sample preparation strategies and analytical platforms (including higher sensitivity NMR systems with possibilities for on-line MS hyphenation) will enhance the relevance of metabolomics in food research. Comprehensive multidimensional techniques, such as GCxGC or LCxLC, are also a revolutionary improvement in separation techniques whose use is expected to grow in Foodomics in the near future. They not only provide enhanced resolution and a huge increase in the peak number but also an increase in selectivity and sensitivity in comparison with conventional separation techniques. Also, capillary electrophoretic techniques and their coupling to mass spectrometry (CE-MS) are ideal tools for metabolomics, due to they do not require extensive sample preparation, their wide range of applications, great efficiency and resolution, and low sample consumption. Besides, CE-MS allows the identification of highly polar and charged metabolites that are difficult to separate by standard LC or GC methods. Metabolomics has many challenges to address regarding the development and grow of the available metabolomic-databases since only a small fraction of the total number of metabolites has been identified and included in the databases so far, the majority of naturallyoccurring metabolites still being unknown. Besides, the scope and range of metabolites within normal and pathophysiological states will require that the field of metabolomics make some unifying assumptions and agree on standards for targeted metabolites and conditions of sampling in order to fully realize its potential in the new Foodomics field.

The challenge in the combination of Foodomics and systems biology is not only at the technological level, that, as mentioned above great improvements are being made and expected in the omics tools, but also on the bioinformatics side (data processing, clustering, dynamics, integration of the various omics levels, etc.) that will have to progress for systems biology to demonstrate all its potential in the new Foodomics discipline. ${ }^{44}$ In this regard, much work is still needed to fill the huge gap in the knowledge on many cellular processes and how they take place at different molecular levels. 


\section{ACKNOWLEDGMENT}

M. H. would like to thank MEC for his Ramón y Cajal contract. This work was supported by Projects AGL2011-29857-C03-01 and CONSOLIDER INGENIO 2010 CSD2007-00063 FUNC-FOOD (Ministerio de Educación y Ciencia).

"Supporting Information Available: This material is available free of charge via the Internet at http://pubs.acs.org." 


\section{REFERENCES}

(1) Hamburg, M.A. Science 2011, 331, 987.

(2) Cifuentes, A.; Dugo, P.; Fanali, S. J. Chromatogr. A 2011, 1218, 7385-7386.

(3) Señorans, F.J.; Ibañez, E.; Cifuentes, A. Crit. Rev. Food Sci. Nutr. 2003, 43, 507-526.

(4) Herrero, M.; Simó, C.; García-Cañas, V.; Ibáñez, E.; Cifuentes, A. Mass Spec. Rev. 2012, 31, 49-69.

(5) Ibañez, E.; Cifuentes, A. Crit. Rev. Food Sci. 2001, 41, 413-450.

(6) Dufour, E. Int. J. Dairy Tech. 2011, 64, 153-165.

(7) Herrero, M.; Ibáñez, E.; Cifuentes, A.; Bernal, J. J. Chromatogr. A 2009, 1216, 7110-7129.

(8) Mendiola, J.A.; Herrero, M.; Cifuentes, A.; Ibañez. E. J. Chromatogr. A 2007, 1152, 234246.

(9) Asensio-Ramos, M.; Ravelo-Perez, L. M.; Gonzalez-Curbelo, M. A.; Hernandez-Borges, J.; J. Chromatogr. A 2011, 1218, 7415-7437.

(10) Bicchi, C.; Cagliero, C.; Rubiolo, P.; Flavour Fragrance J. 2011, 26, 321-325.

(11) Herrero, M.; Simo, C.; Garcia-Canas, V.; Fanali, S.; Cifuentes, A. Electrophoresis 2010, $31,2106-2114$.

(12) Tunick, M. H. J. Agr. Food Chem. 2011, 59, 1477-1480.

(13) Pareja, L.; Fernandez-Alba, A. R.; Cesio, V.; Heinzen, H. TrAC, Trends Anal. Chem. 2011, 30, 270-291.

(14) LeDoux, M. J. Chromatogr. A 2011, 1218, 1021-1036.

(15) Mohamed, R.; Guy, P.A. Mass Spec. Rev. 2011, 30, 1073-1095.

(16) Hajslova, J.; Cajka, T.; Vaclavik, L. TrAC, Trends Anal. Chem. 2011, 30, 204-218.

(17) Agrawal, G. K.; Jwa, N.S.; Rakwal, R. Proteomics 2009, 9, 935-963.

(18) Shepherd, L.V.T.; Fraser, P.; Stewart, D. Bioanalysis 2011, 3, 1143-1159.

(19) García-Cañas, V.; González, R.; Cifuentes, A. TrAC, Trends Anal. Chem. 2004, 23, 637643.

(20) Antignac, J. P.; Courant, F.; Pinel, G.; Monteau F.; Le Bizec, B. TrAC, Trends Anal. Chem. 2011, 30, 292-301.

(21) European Food Safety Agency. 2006. Guidance document of the scientific panel on genetically modified organisms for the risk assessment of genetically modified plants and derived food and feed. Parma: EFSA Communications Department.

(22) Lancova, K.; Dip, R.; Antignac, J.P.; Bizec, B.; le Elliott, C.T.; Naegeli, H. TrAC, Trends Anal. Chem. 2011, 30, 181-191.

(23) Dwivedi, H.P.; Jaykus, L.A. Crit. Rev. Microbiol. 2011, 37, 40-63.

(24) LeDoux, M. J. Chromatogr. A 2011, 1218, 1021-1036.

(25) Faeste, C.K.; Ronning, H.T.; Christians, U.; Granum, P.E. J. Food Prot. 2011, 74, 316-345. 
(26) Raz, S.R.; Haasnoot, W. TrAC, Trends Anal. Chem. 2011, 30, 1526-1537.

(27) Atalay, Y.T.; Vermeir, S.; Witters, D.; Vergauwe, N.; Verbruggen, B.; Verboven, P.; Nicolai, B.M.; Lammertyn, J. Trends Food Sci. Technol. 2011, 22, 386-404.

(28) Schrader, C.; John, R.; Scheilke, A.; J. Food Safety Food Quality 2011, 62, 36-51.

(29) Kantiani, L.; Llorca, M.; Sanchis, J.; Farre, M.; Barcelo, D. Anal. Bioanal. Chem. 2010, $398,2413-2427$.

(30) Magnuson, B.A.; Jonaitis, T.S.; Card, J.W. J. Food Sci. 2011, 76, R126-R133.

(31) Armenta, S.; Garrigues, S.; de la Guardia, M. TrAC, Trends Anal. Chem. 2008, 27, 497511.

(32) Wittwer, J.; Rubio-Aliaga, I.; Hoeft, B.; Bendik, I.; Weber, P.; Daniel, H. Mol. Nutr. Food Res. 2011, 55, 341-358.

(33) Williams, C.M.; Ordovas, J.M.; Lairon, D.; Hesketh, J.; Lietz, G.; Gibney, M.; van Ommen, B. Genes Nutr. 2008, 3, 41-49.

(34) Smith, C.E.; Ordovas, J.M.; Sanchez-Moreno, C.; Lee, Y.C.; Garaulet, M.; Int. J. Obesity 2012, 36, 130-136.

(35) Corella, D.; Arnett, D.K.; Tucker, K.L.; Kabagambe, E.K.; Tsai, M.; Parnell, L.D.; Lai, C.Q.; Lee, Y.C.; Warodomwichit, D.; Hopkins P.N.; Ordovas, J.M. J. Nutrit. 2011, 141, 22192225 .

(36) Kau, A.L.; Ahern, P.P.; Griffin, N.W.; Goodman, A.L.; Gordong, J.I. Nature 2011, 474, 327-336.

(37) Soni, K.A.; Nannapaneni, R.; Tasara, T. Foodborne Pathog. Dis. 2011, 8, 843-852.

(38) García-Cañas, V.; Simó, C.; León, C.; Ibáñez, E.; Cifuentes, A. Mass Spec. Rev. 2011, 30, 396-416.

(39) O'Flaherty, S.; Klaenhammer, T.R. Ann. Rev. Food Sci. Tech. 2011, 2, 353-371.

(40) Hertog, M.L.; Rudell, D.R.; Pedreschi, R.; Schaffer, R.J.; Geeraerd, A.H.; Nicolai, B.M.; Ferguson, I. Postharv. Biol. Tech. 2011, 62, 223-237.

(41) Ibáñez, C.; Valdés, A.; García-Cañas, V.; Simó, C.; Celebier, M.; Rocamora, L.; Gómez, A.; Herrero, M., Castro, M.; Segura-Carretero, A.; Ibáñez, E.; Ferragut, J.A.; Cifuentes A. J. Chromatogr. A (http://dx.doi.org/10.1016/j.chroma.2012.06.008).

(42) Hajslova, J.; Cajka, T.; Vaclavik, L. TrAC, Trends Anal. Chem. 2011, 30, 204-218.

(43) Kaspar, S.; Peukert, M.; Svatos, A.; Matros, A.; Mock, H. Proteomics 2011, 11, 18401850 .

(44) Gehlenborg, N.; O'Donoghue, S.L.; Baliga, N.S. Nat. Methods 2010, 7, S56-S68. 
Carolina Simó is a Tenured Researcher at the National Research Council of Spain (CSIC) in Madrid (Spain). Her running research lines include: i) Development and application of advanced capillary electromigration techniques; ii) Chiral analysis for food quality evaluation; iii) Proteomics and Metabolomics, developing new CE/LC-MS-based approaches.

Virginia García-Cañas is a Tenured Researcher at the National Research Council of Spain (CSIC) in Madrid. She develops her scientific activities in the Laboratory of Foodomics. Her activities include the development of advanced analytical methods for Genomics and Transcriptomics in food quality, safety and Nutrigenomic studies.

Miguel Herrero is a contracted researcher under the "Ramón y Cajal" programme at the Spanish National Research Council (CSIC), in Madrid, Spain. His main research interests are aimed to the study and characterization of new functional ingredients including the development of new advanced extraction and analytical methods to obtain and characterize interesting foodrelated compounds.

Elena Ibáñez: is a Full Professor at the Institute of Food Science Research from the National Council of Spain (CSIC) in Madrid. Her activity includes the development of environmentally clean processes, mainly based on the use of compressed fluids, for food ingredients production and advanced analytical techniques for food ingredients' characterization. She is the Secretary of the Spanish Society of Compressed Fluids (Flucomp), the Vice-president of the Spanish Society of Chromatography and Related Techniques (SEcYTA) and the Delegate from Spain in the Domain of Food and Agriculture in COST Actions.

Alejandro Cifuentes is a Full Research Professor at the National Research Council of Spain (CSIC) in Madrid and Head of the Laboratory of Foodomics. His activity includes advanced analytical methods development for Foodomics, food quality and safety, as well as isolation and characterization of biologically active natural products. He is Editor of TrAC and Electrophoresis and member of the Editorial Board of 12 international journals. 